\title{
ARTÍCULO DE REVISIÓN \\ Microcefalia antes de la llegada del virus Zika: una revisión de tema
}

Fecha de recibido: 25 de marzo de 2019

Fecha de aprobación: 13 de agosto de 2019
Forma de citar este artículo: Marín MJ, Marín AE. Microcefalia antes de la llegada del virus Zika: una revisión de tema. Med UPB. 2020;39(1):42-48. DOI:10.18566/medupb.v39n1.a07

1 Facultad de Medicina, Universidad Pontificia Bolivariana. Medellín, Colombia.

2 Universidad Militar Nueva Granada. Medellín, Colombia.

Dirección de

correspondencia: María José Marín Castro. Correo electrónico: maria.marinc@ upb.edu.co

\author{
Microcephaly before the arrival of the Zika virus: A review of \\ the topic / Microcefalia antes da chegada do vírus Zika: uma \\ revisão do assunto
}

María José Marín Castro ${ }^{1}$, Andrés Eduardo Marín Castro ${ }^{2}$

\begin{abstract}
RESUMEN
Los reportes de casos de microcefalia en Brasil han significado un reto para la salud pública, especialmente en países de Latinoamérica como Colombia, pues a pesar de su presunta relación con el virus Zika, se desconoce cómo los otros factores implicados en la etiología de la microcefalia se relacionan con este aumento de casos. También se cuenta con pocas estadísticas epidemiológicas acerca del comportamiento de los factores etiológicos de microcefalia hasta el año clave de 2015, no solo en Brasil sino en la mayoría de países latinoamericanos. Las estimaciones de la incidencia y prevalencia de microcefalia son variables debido a diferencias en las definiciones, estándares de seguimiento y diferencias entre las poblaciones que tienen y las que no tienen validados los estándares de diagnóstico y seguimientos de microcefalia. Se realiza una revisión con una mirada general a la microcefalia, se unifican definiciones, clasificación y etiología. Palabras Clave: microcefalia; virus Zika; etiología
\end{abstract}

\section{ABSTRACT}

The reports of cases of microcephaly in Brazil have posed a challenge for the public health, in particular, in Latin American countries such as Colombia, since despite their alleged relationship with the Zika virus, there is no information on the relation of other factors involved in the etiology of microcephaly are related with this increase in cases. There are also very few epidemiological statistics on the behavior of the etiological factors of microcephaly until the crucial year of 2015, not only in Brazil, but also in most Latin American countries. The changes in the incidence and prevalence of microcephaly are very variable due to the dissimilarities in the definitions, the development criteria and the differences among the populations with and without certified diagnostic standards and follow-up criteria for microcephaly. A general review of the microcephaly issue is made, in order to unify definitions, classification and etiology.

Keywords: microcephaly; Zika Virus; etiology

\section{RESUMO}

Os relatórios de casos de microcefalia no Brasil há significado um desafio para a saúde pública, especialmente nos países da América Latina como a Colômbia, pois a pesar de sua suposta relação com o vírus Zika, se desconhece como os outros fatores implicados na etiologia da microcefalia se relacionam com este aumento de casos. Também se conta com poucas estadísticas epidemiológicas sobre o comportamento dos fatores etiológicos de microcefalia até o ano chave de 2015, não só no Brasil se não na maioria de países latino-americanos. As estimações da incidência e prevalência de microcefalia 
são variáveis devido a diferenças nas definições, padrões de seguimento e diferenças entre as populações que têm e as que não têm validados os padrões de diagnóstico e seguimentos de microcefalia. Se realiza uma revisão com uma olhada geral à microcefalia, se unificam definições, classificação e etiologia.

Palavras Chave: microcefalia; vírus Zika; etiologia

\section{INTRODUCCIÓN}

Los reportes de aumento en el número de casos de microcefalia en Brasil han significado un reto para la salud pública, especialmente en Latinoamérica. Se cuenta con pocas estadísticas epidemiológicas sobre el comportamiento de los factores etiológicos de la microcefalia hasta el momento clave de la alerta epidemiológica (diciembre de 2015), no solo en Brasil sino en gran parte de Latinoamerica ${ }^{1-6}$.

Antes de 2015, el número anual de casos de microcefalia en Brasil estaba por debajo de 200. Entre mediados de 2015 y el 30 de enero de 2016 se reportaron 4783 casos sospechosos de microcefalia, incluyendo recién nacidos y pérdidas fetales. De estos, 1103 se estudiaron y 404 (36.2\%) fueron clasificados como casos confirmados de microcefalia. Entre los confirmados, las anomalías cerebrales fueron detectados por imágenes en 387 bebés y se detectó el virus Zika en 17 bebés, incluidas dos pérdidas fetales ${ }^{6}$. Parece ser una alta tasa de verdaderos positivos, sin embargo, hay que ser cautelosos. Este aumento temporal de presuntos casos de microcefalia podría estar distorsionado por el subregistro prexistente, la mayor conciencia con la evaluación y notificación de los niños y el cambio de la definición de la microcefalia en el tiempo ${ }^{7-12}$.

La posibilidad de un exceso de informes y diagnósticos erróneos fue planteada por la Red de Malformaciones Congénitas de América Latina, lo que dio lugar a la especulación en la prensa científica sobre la magnitud del aumento de casos de microcefalia $^{6,13}$.

A pesar de los inconvenientes en el registro de casos, no cabe duda que la microcefalia en recién nacidos en América latina, especialmente en Brasil, afecta el sistema de salud global. Con esta revisión buscamos caracterizar la microcefalia, resaltar sus aspectos más importantes e impactar en el desarrollo de una buena estrategia de tamización para recién nacidos, así como en un diagnóstico y tratamiento oportunos para los ya afectados.

\section{Definiciones}

La medición de la circunferencia de la cabeza, también llamada perímetro cefálico (PC) o circunferencia occipitofrontal (COF), es útil para el cribado de microcefalia, independientemente de la causa ${ }^{6}$. La Academia Americana de Pediatría (AAP) recomienda que todos los niños tengan una medición en cada visita de niño sano, hasta los dos años de edad ${ }^{7}, o$ ante la presencia de signos neurológicos. Las desviaciones del crecimiento normal de la cabeza puede ser la primera indicación de un problema congénito, genético o adquirido ${ }^{8-13}$.

La microcefalia está incluida en el grupo de: malformaciones congénitas, deformaciones y aberraciones cromosómicas, de la décima revisión de la Clasificación Internacional de Enfermedades (CIE -11). Se define como un signo clínico, en el que el PC es dos a tres desviaciones estándar (DE) menor que el promedio para el edad y sexo. En algunos casos se asocia con alteraciones en la estructura del encéfalo y el desarrollo neurológico. Si no hay anomalías estructurales no se considera una anomalía mayor.

Los valores de referencia del perímetro cefálico varían según la edad gestacional al nacer y el sexo ${ }^{1}$ y permiten clasificar la microcefalia de la siguiente manera ${ }^{13-20}$ :

- Microcefalia leve o limítrofe: PC entre dos y tres DE por debajo de la media.

- Microcefalia moderada: PC entre tres y cinco DE por debajo de la media.

- Microcefalia grave: PC inferior a cinco

DE por debajo de la media. 
Sin embargo, la definición de microcefalia es controvertida $^{18,21}$. Algunos autores la definen como un PC de dos DE por debajo de la media (es decir, inferior al percentil 3), otros indican que se debe ajustar, según sea necesario, a la edad gestacional o $\mathrm{PC}$ de los padres ${ }^{22}$. Otros definen la presencia de microcefalia en todo paciente con un PC de más de $3 \mathrm{DE}$ por debajo de la media ${ }^{19}$.

El consenso establecido por la Academia Americana de Neurología (AAN) define microcefalia como 2 DE por debajo de la media ${ }^{23}$.

En la alerta epidemiológica emitida el primero de diciembre de 2015, la Organización Panamericana de la Salud (OPS) y la Organización Mundial de la Salud (OMS) recomendaban utilizar como definición transitoria un límite de inclusión de $-2 \mathrm{DE}$, sin embargo, recientemente recomiendan considerar como criterio de microcefalia congénita un valor por debajo del percentil tres, según curvas de referencia específicas, dependiendo de la edad gestacional y del sexo. Así mismo, sugieren consignar el valor absoluto de $\mathrm{PC}$ en centímetros con un decimal, junto con el valor de percentil correspondiente, como se muestra en la Tabla $1^{1,24,25}$.

Tabla 1. Valores absolutos de las definiciones de microcefalia OPS/OMS.

\begin{tabular}{lcc}
\hline Límite de inclusión & Niñas & Niños \\
\hline - 2 DE & $31.5 \mathrm{~cm}$ & $31.9 \mathrm{~cm}$ \\
Percentil 3 & $31.6 \mathrm{~cm}$ & $32.0 \mathrm{~cm}$ \\
\hline
\end{tabular}

Dado que el crecimiento de la cabeza es impulsado por el crecimiento cerebral, la microcefalia implica generalmente microencefalia (cerebro anormalmente pequeño, diagnosticado por neuroimagen o diagnóstico neuropatológico), no obstante, la microencefalia puede estar presente en niños con $\mathrm{PC}$ normal ${ }^{24,25}$.

Como se mencionó, los valores deben estandarizarse según la edad gestacional. Para los recién nacidos a término se sugiere utilizar las curvas de crecimiento de la OMS, según sexo. En el caso de recién nacidos prematuros se deben utilizar referencias específicas (Fenton ${ }^{26}$, Estudio Intergrowth ${ }^{27}$, entre otros) según edad gestacional y sexo.

Entre 2001 y 2008 se llevó a cabo un estudio de cohorte retrospectivo en 33 centros de atención primaria pediátrica de los Estados Unidos que mostró que las curvas de referencia de PC para el control y la prevención de enfermedades (CDC) de ese país y las de la OMS, tienen diferencias entre ellas y con los datos de la población evaluada, especialmente en niños con PC mayores ${ }^{19}$.

Es importante evaluar cómo han evolucionado los criterios de la circunferencia de la cabeza para definir un caso de microcefalia. En la Tabla 2 se exponen los más aceptados recientemente como definitorios de microcefalia ${ }^{1-3,13,21,25,27}$.

La propuesta del Ministerio de Salud de Brasil, hasta diciembre de 2015, de utilizar un punto de corte fijo para los nacidos en la franja de semanas que implican un parto a término no es adecuada ya que, se debe tener en cuenta que muchos nacimientos a término ocurren antes de las 39 semanas de gestación y que las tasas de cesárea a edades gestacionales consideradas a término son considerablemente altas. Además, las razones para elegir la referencia Fenton ${ }^{24}$ no son claras, las curvas de Fenton se basan en un metaanálisis de seis estudios en países desarrollados con métodos no estandarizados ${ }^{12,28,29}$.

En un análisis reciente realizado ante el aumento del numero de casos de microcefalia en Brasil, presunta-

Tabla 2. Criterios definitorios de microcefalia.

\begin{tabular}{|c|c|c|c|}
\hline Fuente & Límite de inclusión & S\% & E\% \\
\hline $\begin{array}{l}\text { Ministerio de Salud de } \\
\text { Brasil (Hasta diciembre } \\
\text { 2015) }\end{array}$ & $\begin{array}{l}\leq 33 \mathrm{~cm} \text { para recién nacidos a término, de ambos sexos o }<-2 \text { de } \mathrm{DE} \\
\text { o percentil } 3\left(\text { Fenton }{ }^{21} \text { ) para recién nacidos pretérmino, según edad }\right. \\
\text { gestacional y sexo }\end{array}$ & 92 & 79.3 \\
\hline $\begin{array}{l}\text { Ministerio de salud de } \\
\text { Brasil (Desde diciembre } \\
\text { 2015)2 }\end{array}$ & $\begin{array}{l}\leq 32 \mathrm{~cm} \text { para recién nacidos a término de ambos sexos o }<-2 \text { de } D E \\
\text { o percentil } 3\left(\text { Fenton }{ }^{21} \text { ) para recién nacidos pretérmino, según edad }\right. \\
\text { gestacional y sexo }\end{array}$ & 86 & 93.8 \\
\hline Estudio InterGrowth ${ }^{27}$ & $\leq 3 \mathrm{DE}$ según edad gestacional y sexo para todos los recién nacidos & 57 & 99.9 \\
\hline +Estudio InterGrowth ${ }^{27}$ & $\leq 2 \mathrm{DE}$ según edad gestacional y sexo para todos los recién nacidos & 85 & 97.8 \\
\hline$+\mathrm{OPS} \mathrm{OMS}^{1}$ & $\begin{array}{l}\text { < Percentil } 3(\mathrm{OMS}) \text { para recién nacidos a término }(<31.6 \mathrm{~cm} \text { para niñas } \\
\text { y } 32 \mathrm{~cm} \text { para niños) y referencias de crecimiento Fenton }{ }^{25} \text { o InterGrowt }{ }^{27} \\
\text { para recién nacidos pretérmino }{ }^{25}\end{array}$ & 80 & 96.1 \\
\hline
\end{tabular}

+ criterios recomendados / S: Sensibilidad / E: Especificidad 
mente por el virus Zika, se aplicó el estándar propuesto por el estudio InterGrowth. En este estudio prospectivo multicéntrico en ocho países, las había una estimación confiable de la edad gestacional, las mediciones antropométricas de los recién nacidos se realizaron por el mismo equipo y se excluyeron los casos de microcefalia. En este trabajo se determinó un punto de corte con mayor especificidad para definir un caso de microcefalia. Se propuso entonces, un punto de corte de $-3 \mathrm{DE}$ debajo del valor medio para el PC del recién nacido, sobre la base de que la mayoría de recién nacidos con PC entre -2 DE y -3 $\mathrm{DE}$ no tienen evidencia de malformaciones ${ }^{12}$. Este punto de corte ha sido utilizado tradicionalmente para estudios de malformaciones congénitas ${ }^{4}$.

Como se mencionó anteriormente, la OMS define microcefalia como $\mathrm{PC}$ por debajo de $-2 \mathrm{DE}$, según sexo y edad gestacional. Pero sólo si hay anomalías estructurales del cerebro. La Organización afirma explícitamente que la ausencia de tales anomalías descarta el diagnóstico $^{1,25,30}$.

A esta diferencia de criterios se suma el desequilibrio entre la especificidad y la sensibilidad estadística. Dado que no hay un tratamiento eficaz para la microcefalia congénita, hay un fuerte argumento para dar prioridad a la especificidad sobre la sensibilidad. El aumento de la especificidad reduciría el potencial iatrogénico de la radiación durante la tomografía cerebral u otra neuroimagen, ayudaría a aliviar los efectos emocionales en los padres y reduciría la carga y los costes del sistema de salud ya sobrecargado. Los argumentos para dar prioridad a la sensibilidad incluyen que estamos en las primeras etapas de una "nueva epidemia" de la que poco se sabe y que los bebés con microcefalia que se beneficiarían de la estimulación intelectual temprana podrían ser exactamente aquellos cuya circunferencia de la cabeza está más cerca del rango normal ${ }^{12}$.

En resumen, se recomienda el uso de un conjunto coherente de criterios de diagnóstico para la sospecha de microcefalia, que tengan en cuenta la edad gestacional de los recién nacidos a término y pretérmino. Estas La sensibilidad de un punto de corte de $-3 \mathrm{DE}$ para el PC de parecer demasiado baja, en particular si durante una posible nueva epidemia no se quieren perder muchos casos. Se propone entonces el punto de corte de $\leq-2 \mathrm{DE}$, que tiene una sensibilidad similar a las demás recomendaciones, pero mejor especificidad, lo que evita sobrestimar el número real de casos (es decir, evita incluir niños normales con cabezas pequeñas), contrariamente a lo ocurrido en Brasil con los presuntos casos por virus $\mathrm{Zika}^{12}$.

También es importante que los trabajadores de salud midan la circunferencia de la cabeza en todos los recién nacidos mediante técnicas antropométricas estandarizadas. Una mejor medición y el uso de los estándares de crecimiento adecuados son esenciales para la vigilancia continua de los casos microcefalia. Hay disponibles varias curvas de crecimiento del PC. En 1977, una referencia de cómo estaban creciendo los niños fue publicada por el Centro Nacional de Estadísticas de Salud (NCHS) de los Estado Unidos ${ }^{31}$. En el 2000, el CDC publicó una referencia en reemplazo a la interior, debido a la preocupación de que las curvas originales no eran representativas de la población, estas curvas de referencia se recomiendan actualmente para su uso en los Estados Unidos ${ }^{27}$. En 2006, la OMS publicó un estándar de crecimiento basado en datos de niños de seis países ${ }^{32,33}$. En la Tabla 3 se referencias las curvas para el seguimiento del PC desde los 0 hasta los 18 años $7,22,29-32,34-38$. Todas difieren unas de otras y en las recomendaciones de su aplicación de acuerdo con la edad, lo que afecta la clasificación y el seguimiento del PC en las diversas poblacionales, etnias $y \operatorname{razas}^{13,34-37 .}$

Tabla 3. Curvas de referencia para el seguimiento del PC.

\begin{tabular}{lc}
\hline Fuente & Rango de edades \\
\hline CDC & $0-36$ meses* $^{*}$ \\
OMS & $0-5$ años* $^{*}$ \\
Nellhaus, metanalisis & $0-18$ años \\
internacional $^{38}$ & \\
$\begin{array}{l}\text { Fels, estudio longitudinal de 888 } \\
\text { niños blancos de EE. UU.22 }\end{array}$ & $0-18$ años \\
\hline
\end{tabular}

*En septiembre de 2010, el CDC recomienda que las curvas de la OMS se utilicen de 0 a 2 años y las del CDC para niños mayores de 2 años ${ }^{35}$.

Técnica de medición: la cinta métrica debe rodear la cabeza y abarcar la superficie entre 1 a $2 \mathrm{~cm}$ por encima de la glabela en la parte anterior y la parte más prominente del occipucio en la parte posterior. La medición en el recién nacido puede ser poco fiable hasta el tercer o cuarto día de vida, ya que puede verse afectada por el moldeamiento de la cabeza durante el parto o cefalohematoma. En lactantes la medición puede verse afectada por el pelo grueso y la deformación o la hipertrofia del hueso craneal.

\section{Clasificación}

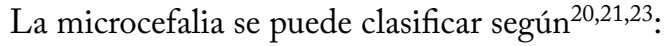

- Tiempo de presentación

- Primaria: también llamada congénita, se presentan en general al nacimiento y en estos casos el cerebro es pequeño debido a malformaciones congénitas. 
- Secundaria: también llamada posnatal, se refiere a una desviación del crecimiento normal en un cerebro que era de tamaño normal al nacer, que sufre un daño, por lo general en el periodo perinatal o posnatal. Una circunferencia normal seguida de un fallo en el crecimiento, indica casi siempre una microcefalia de este tipo.

- Etiología: genética o ambiental ${ }^{21}$.

- Relación con otros parámetros de crecimiento ${ }^{21}$ :

- Simétrica o proporcional: cuando la disminución del $\mathrm{PC}$ es $>2$ a 3 DE de la media, pero proporcional al peso y la talla.

- Asimétrica o desproporcionada.

- Por asociación con otras anomalías ${ }^{21}$ :

- Aislada o pura: no asociada con ninguna otra anomalía.

- Sindrómatica o compleja: se asocia con una o más anomalías.

\section{Patogénesis}

La microcefalia tiene dos mecanismos principales ${ }^{11,38,39}$ :

- La falta de desarrollo o desarrollo anormal del cerebro relacionada con una noxa durante el período de formación embriológica.

- Lesión o noxa a un cerebro previamente normal, este tipo de microcefalia es el resultado de una reducción en el número conexiones sinápticas.

Durante la gestación es importante evaluar e intervenir sobre los factores de riesgo relacionados con la microcefalia, tales como enfermedades infecciosas, nutrición inadecuada, exposición a alcohol, tabaco y ciertos medicamentos, y de este modo prevenir su desarrollo. También se debe procurar mantener los cuidados adecuados del neonato, que ayudan a su correcto desarrollo ${ }^{11,38,39}$.

\section{Etiología}

Diversas anomalías genéticas y agresiones ambientales afectan el desarrollo del cerebro ${ }^{11,24}$. En una serie de 680 niños con microcefalia, la distribución etiológica se comportó asi $1^{11,40}$ :

- Genética o presumiblemente genético (aberraciones cromosómicas, enfermedades monogénicas, síndromes genéticos): $29 \%$.

- Lesión cerebral prenatal y perinatal (exposición a teratógenos, enfermedad materna, complicaciones del parto): $27 \%$.

- Craneosinostosis: $2 \%$.
- Lesión cerebral postnatal (infección, infarto, encefalitis, traumatismo): $2 \%$

- Etiología desconocida (probablemente genética): $41 \%$

La mayoría de los pacientes de esta serie tenía signos neurológicos o neuropsiquiátricos (discapacidad intelectual, epilepsia) ${ }^{34}$. Es probable que estas causas sean diferentes a las encontradas en centros de atención primaria $^{11,41-45}$.

Las anomalías neuroanatómicas asociados con microcefalia incluyen defectos del tubo neural, holoprosencefalia, aprosencefalia, lisencefalia, esquizencefalia, polimicrogiria, entre otras ${ }^{11,41-45}$.

Varios trastornos metabólicos pueden estar asociados con la microcefalia, pero la prevalencia de estos en los niños con microcefalia es baja (1\% a 5\%). Incluyen aminoacidurias (fenilcetonuria, aciduria metilmalónica), trastornos del ciclo de la urea (citrulinemia) y enfermedades de depósito ${ }^{10-13,23}$.

Los factores ambientales que pueden reducir el tamaño cerebral incluyen ${ }^{19,42-50}$ :

- Infecciones del sistema nervioso central (prenatales, perinatales y posnatales). En donde se incluyen las infecciones que clásicamente se han agrupado en el acrónimo TORCH (T: toxoplasmosis, R: rubeola, C: citomegalovirus y $\mathrm{H}$ : herpes. La O correspondería a otras infecciones entre las que inicialmente se incluyeron varicela y sífilis, pero que en la actualidad pueden englobar parvovirus B19, papilomavirus, malaria y tuberculosis e infección por virus Zika).

- Exposición intrauterina a dogas y toxinas.

- Encefalopatía hipóxico isquémica.

- Malnutrición.

- Enfermedades sistémicas.

\section{CONCLUSIÓN}

Los datos sobre incidencia y prevalencia de microcefalia son variables debido a diferencias en las definiciones, diferentes estándares de seguimiento, cambios de estos últimos en el tiempo y diferencias entre las poblaciones que tienen y las que no tienen validados estándares de diagnóstico y seguimientos. Además, no resulta clara la asociación con la virus Zika. La Red de Malformaciones Congénitas de América Latina planteó la posibilidad de un exceso de diagnósticos erróneos y su informe dio lugar a la especulación en la prensa científica sobre la verdadera magnitud del aumento de casos de microcefalia. 


\section{DECLARACIÓN DE CONFLICTO DE INTERESES:}

Los autores declaran no tener ningún conflicto de intereses.

\section{REFERENCIAS}

1. Organización Panamericana de la Salud. Lineamientos preliminares de vigilancia de microcefalia en recién nacidos en entornos de riesgo de circulación de virus Zika. Recuperado a partir de: http://www.paho.org/hq/index.php?option=com_docman\&task=doc_ view\&Itemid=270\&gid=32999.

2. Brasil Ministério da Saúde. Secretaria de Vigilância em Saúde. Departamento de Vigilância das Doenças Transmissíveis. Protocolo de vigilância e resposta à ocorrência de microcefalia relacionada à infecção 16 pelo vírus Zika. Brasília: Ministério da Saúde, 2015.

3. Brasil Ministério da Saúde. Secretaria de Vigilância em Saúde. Departamento de Vigilância Note Informativa 01/2015. COES 14 Microcefalias. Brasília: Ministério da Saúde, 2015.

4. Latin American Network of Congenital Malformations. Microcefalias en el ECLAMC y en Brasil. 2015. http://www.eclamc.org/ microcefaliaarchivos.php.

5. Almeida IM, Ramos CV, Rodrigues DC, Sousa AC, Nascimento ML, Silva MV, et al. Clinical and epidemiological aspects of microcephaly in the state of Piauí, northeastern Brazil, 2015-2016. J Pediatr (Rio J). 2018; 95(4):466-74.

6. Díaz-Menéndez M, Trigo E, De la Calle-Prieto F, Arsuaga M. Zika virus infection during the Olympic Games in Rio: A fear or an actual risk? Rev Clin Esp. 2017; 217(3):155-60.

7. Caylà JA, Domínguez Á, Rodríguez E, De Ory F, Vázquez A, Fortuny C. [Zika virus infection: A new public health emergency with great media impact]. Gac Sanit. 2016; 30(6):468-71.

8. Nunes ML, Carlini CR, Marinowic D, Neto FK, Fiori HH, Scotta MC, et al. Microcephaly and Zika virus: A clinical and epidemiological analysis of the current outbreak in Brazil. J Pediatr (Rio J). 2016; 92(3):230-40.

9. Mlakar J, Korva M, Tul N, Popovic M, Polj`sak-Prijatelj M, Mraz J, et al. Zika virus associated with microcephaly. N Engl J Med. 2016; 374:951-8.

10. Martínez P, Suy A, Sánchez-Montalvá A, Rodó C, Salvador F, MolinaI. Zika fever. Enferm Infecc Microbiol Clin. 2016; 34(4):247-52.

11. Boom JA. Microcephaly in infants and children: Etiology and evaluation. [Internet]. 2016. Recuperado a partir de: http://www.uptodate.com.consultaremota.upb.edu.co/contents/ microcephaly-in-infants-and-children-etiology-and-evaluation?source=search_result\&searc $\mathrm{h}=$ microcephaly\&selectedTitle=1\%7E135.

12. Chenel C, Wood C, Gourrier E, Zittoun J, Casadevall I, Ogier H. [Neonatal haemolytic-uraemic syndrome, methylmalonic aciduria and homocystinuria caused by intracellular vitamin B 12 deficiency. Value of aetiological diagnosis]. Arch Fr Pediatr. 1993; 50:749-54.

13. Victora CG, Schuler-Faccini L, Matijasevich A, Ribeiro E, Pessoa A, Barros FC. Microcephaly in Brazil: How to interpret reported numbers? Lancet. 2016; 387(10019):621-4.

14. Daymont $C$, Hwang W-T, Feudtner $C$, Rubin D. Head-circumference distribution in a large primary care network differs from CDC and WHO curves. Pediatrics. 2010; 126(4):e836-42.

15. Stoll $C$. Problems in the diagnosis of fragile $X$ syndrome in young children are still present. Am J Med Genet. 2001; 100(2):110-5.

16. Zvulunov A, Weitz R, Metzker A. Neurofibromatosis type 1 in childhood: Evaluation of clinical and epidemiologic features as predictive factors for severity. Clin Pediatr (Phila). 1998; 37(5):295-9.

17. Furuta T, Tabuchi A, Adachi Y, Mizumatsu S, Tamesa N, Ichikawa T, et al. Primary brain tumors in children under age 3 years. Brain Tumor Pathol. 1998; 15(1):7-12.

18. Tomita T, McLone DG. Brain tumors during the first twenty-four months of life. Neurosurgery. 1985; 17(6):913-9.

19. Gleeson JG, Dobyns WB, Plawner L, Ashwal S. Congenital structural defects. In: Pediatric Neurology Principles and Practice, 4th ed, Swaiman KF, Ashwal S, Ferriero DM (Eds), Mosby Elsevier, Philadelphia 2006. p.399.

20. Abuelo D. Microcephaly syndromes. Semin Pediatr Neurol. 2007; 14(3):118-27.

21. Leroy JG, Frías JL. Nonsyndromic microcephaly: An overview. Adv Pediatr. 2005; 52:261-93.

22. Roche AF, Mukherjee D, Guo SM, Moore WM. Head circumference reference data: Birth to 18 years. Pediatrics. 1987; 79(5):706-12.

23. Ashwal S, Michelson D, Plawner L, Dobyns WB. Practice parameter: Evaluation of the child with microcephaly (an evidence-based review): Report of the Quality Standards Subcommittee of the American Academy of Neurology and the Practice Committee of the Child Neurology Society. Neurology. 2009; 73(11):887-97. 
24. Menkes JH, Sarnat HB, Flores-Sarnat L. Malformations of the central nervous system. In: Child Neurology, 7th ed, Menkes JH, Sarnat HB, Maria BL (Eds), Lippincott Williams \& Wilkins, Philadelphia 2006. p.284.

25. WHO. The WHO Child Growth Standards [Internet]. 2016. Recuperado a partir de: http://www. who.int/childgrowth/en/

26. Fenton TR, Kim JH. A systematic review and meta-analysis to revise the Fenton growth chart for preterm infants. BMC Pediatr. 2013; 13(59):10.1186/1471-2431-13-59.

27. Villar J, Cheikh L, Victora CG. International standards for newborn weight, length, and head circumference by gestational age and sex: the Newborn Cross-Sectional Study of the INTERGROWTH-21st Project. Lancet 2014; 384:857-68.

28. Gibbons L, Belizan JM, Lauer JA, Betran AP, Merialdi M, Althabe F. Inequities in the use of cesarean section deliveries in the world. Am J Obstet Gynecol 2012; 206:e1-19.

29. Giuliani F, Ohuma E, Spada E. Systematic review of the methodological quality of studies designed to create neonatal anthropometric charts. Acta Paediatr 2015; 104:987-96.

30. WHO. Birth defects surveillance: A manual for programme managers. Geneva: World Health Organization, 2014.

31. Hamill PV, Drizd TA, Johnson CL, Reed RB, Roche AF. NCHS growth curves for children birth-18 years. United States. Vital Health Stat. 1977; (165):1-74.

32. Kuczmarski RJ, Ogden CL, Guo SS. 2000 CDC growth charts for the United States: Methods and development. Vital Health Stat. 2002; (246):1-190.

33. WHO, Multicentre Growth Reference Study Group. WHO child growth standards: Head circumference for age, arm circumference for age, triceps skinfold for age and subscapular skinfold for age — Methods and Development. Geneva, Switzerland; 2007.

34. Rollins JD, Collins JS, Holden KR. United States head circumference growth reference charts: Birth to 21 years. J Pediatr. 2010; 156(6):907-13.

35. Grummer-Strawn LM, Reinold C, Krebs NF, Centers for Disease Control and Prevention (CDC). Use of World Health Organization and CDC growth charts for children aged 0-59 months in the United States. MMWR Recomm Rep Morb Mortal. 2010; 59(RR-9):1-15.

36. Wright CM, Inskip HM, Godfrey K, Williams AF, Ong KK. Monitoring head size and growth using the new UK-WHO growth standard. Arch Dis Child. 2011; 96(4):386-8.

37. Natale $V$, Rajagopalan A. Worldwide variation in human growth and the World Health Organization growth standards: A systematic review. BMJ Open. 2014; 4(1):e003735.

38. Nellhaus $\mathrm{G}$. Head circumference from birth to eighteen years. Practical composite international and interracial graphs. Pediatrics. 1968; 41(1):106-14.

39. Woods CG. Human microcephaly. Curr Opin Neurobiol. 2004; 14(1):112-7.

40. Von der Hagen $M$, Pivarcsi $M$, Liebe J. Diagnostic approach to microcephaly in childhood: A two-center study and review of the literature. Dev Med Child Neurol 2014; 56:732.

41. Abuelo D. Microcephaly syndromes. Semin Pediatr Neurol. 2007; 14:118-27.

42. Baxter PS, Rigby AS, Rotsaert MH, Wright I. Acquired microcephaly: Causes, patterns, motor and IQ effects, and associated growth changes. Pediatrics. 2009; 124:590-5.

43. Bartram JL, Rigby AS, Baxter PS. The "Lassoo" tape: stretch ability and observer variability in head circumference measurement. Arch Dis Child. 2005; 90(8):820- 21.

44. Bolduc FV, Shevell MI. Corrected head circumference centiles as a possible predictor of developmental performance in high-risk neonatal intensive care unit survivors. Dev Med Child Neurol. 2005; 47(11):766-77.

45. Nard JA. Abnormal head size and shape. In: Common \& Chronic Symptoms in Pediatrics, Gartner JC, Zitelli BJ (Eds), Mosby, St. Louis 1997.

46. Coronado R, Giraldo J, Macaya A, Roig M. Head circumference growth function as a marker of neurological impairment in a cohort of microcephalic infants and children. Neuropediatrics. 2012; 43:271-4.

47. Holmes LB, Harvey EA, Coull BA. The teratogenicity of anticonvulsant drugs. N Engl J Med. 2001; 344:1132.

48. Dansky LV, Andermann E, Rosenblatt D, Sherwin AL, Andermann F. Anticonvulsants, folate levels, and pregnancy outcome: A prospective study. Ann Neurol. 1987; 21:176-82.

49. Holmes LD, Adams J, Coull B, Harvey EA. Anticonvulsant face: Association with cognitive dysfunction. Pediatric Res 2000; 47: Suppl:82A.

50. Wells PG, Winn LM. Biochemical toxicology of chemical teratogenesis. Clin Rev Biochem Mol Biol. 1996; 31:1-40. 